\title{
Spigelian Hernia: A Cases Report
}

\author{
Y. Dkhissi ${ }^{1 *}$, I. Labbi ${ }^{1}$, H.ElBouhaddouti ${ }^{1}$, O. Mouaqit ${ }^{1}$, E. Benjelloun ${ }^{1}$, A. Ousadden ${ }^{1}$, K.AIT Taleb ${ }^{1}$
}

\author{
${ }^{1}$ Department of general surgery A, CHU Hassan II FES, 30000, Morocco
}

DOI: $10.36347 /$ sasjs.2021.v07i04.002

| Received: 26.02.2021 | Accepted: 22.03.2021 | Published: 02.04.2021

*Corresponding author: Y. Dkhissi

Abstract

Introduction: Spigelian hernias or lateral ventral hernia are rare defects of the abdominal wall usually appearing between the abdominal muscles, lateral to the rectus abdominis and through a debilitated Spigelian aponeurosis. It is a rare clinical entity, accounting for 0.10 to $1 \%$ of hernias. Also, it seemed appropriate to us to report this two cases collected in the surgery department A of the CHU Hassan II of Fez. Observation: We report the observation of two patients:a 84-year-old patient with a history of ischemic heart disease stented under treatment, renal failure and arterial hypertension who presented a left paraumbilical swelling gradually increasing in size, and a 30-year-old man admitted to the emergency room after a fall from the 2nd floor following an electrification ,Spiegel hernia was suspected on clinical examination, and the diagnosis of left anterolateral eventration was retained on abdominal computed tomography. A hernia repair by prolene mesh and parietal raffia were performed and the postoperative follow-up was simple. Conclusion: Spiegelian hernia is a rare affection; its clinical diagnosis can be difficult. It is asymptomatic in $90 \%$ of cases and its positive diagnosis is radiological. The significant risk of strangulation requires surgical treatment once the diagnosis is confirmed.

Keywords: Spiegelian hernia, strangulation, mesh repair.

Copyright $(92021$ The Author(s): This is an open-access article distributed under the terms of the Creative Commons Attribution 4.0 International License (CC BY-NC 4.0) which permits unrestricted use, distribution, and reproduction in any medium for non-commercial use provided the original author and source are credited.

\section{INTRODUCTION}

Spigelian hernia is a hernia of the anterolateral abdominal wall, which is defined by the protrusion of a hernial sac through an anatomical orifice acquired or congenital from the Spiegel line. It develops in an interparietal situation between the large muscles of the abdomen [1]. It may contain omentum, small bowel, and colon, and its contents may or may not be reducible. It is relatively rare, and represents about $2 \%$ of the total anterior parietal hernias and is associated with a high risk of strangulation $[1,2]$.

His positive diagnosis is radiological and surgery is his only treatment. In the light of an observation and a review of the literature, the aim of our work is to highlight the diagnostic difficulties and the different therapeutic modalities of this pathology.

\section{CASES PRESENTATION}

\section{Case 1}

This was a 84-year-old man with a history of ischemic heart disease stented under treatment, renal failure and arterial hypertension, presenting for 3 years a left pararectal mass subumbilical, gradually increasing in size, without transit disorder and without deterioration of the general condition. On examination, it was a mass of about $5 \mathrm{~cm}$ in diameter, soft, painless, impulsive to cough and reducible, with no inflammatory sign, suggesting primarily lipomatous proliferation. Ultrasound had suspected a Spigelian hernia; diagnostic confirmation was obtained by abdominal CT (figure 1 ) which showed subumbilical muscle dehiscence at the level of the semilunar line of Spiegel with the presence of the hernial sac containing the greater omentum.

Surgical exploration found a Spigelian hernia containing viable omentum, treatment consisted of reduction of the hernia with wall repair by nonabsorbable mesh, and the postoperative consequences were simple.

\section{Case 2}

This was a 30-year-old man with no previous history, victim of a fall from the 2nd floor following an electrification ,physical examination revealed a stable patient with painful, firm and irreducible mass of $7 \times 3$ $\mathrm{cm}$ located in the right lower quadrant. The rest of the examination was normal. The diagnostic confirmation was obtained by the abdominal CT scan, which showed muscle dehiscence in the sub umbilical level at the semi lunar Spigelian line with the hernia sac containing small bowel and epiploon without signs of suffering (figure 2). 
Surgical exploration found a Spigelian hernia containing viable small bowel, treatment consisted of reduction of the hernia with parietal raffia using interrupted suture, and .The postoperative course was uneventful. The patient had a favorable outcome for ten months without recurrence.

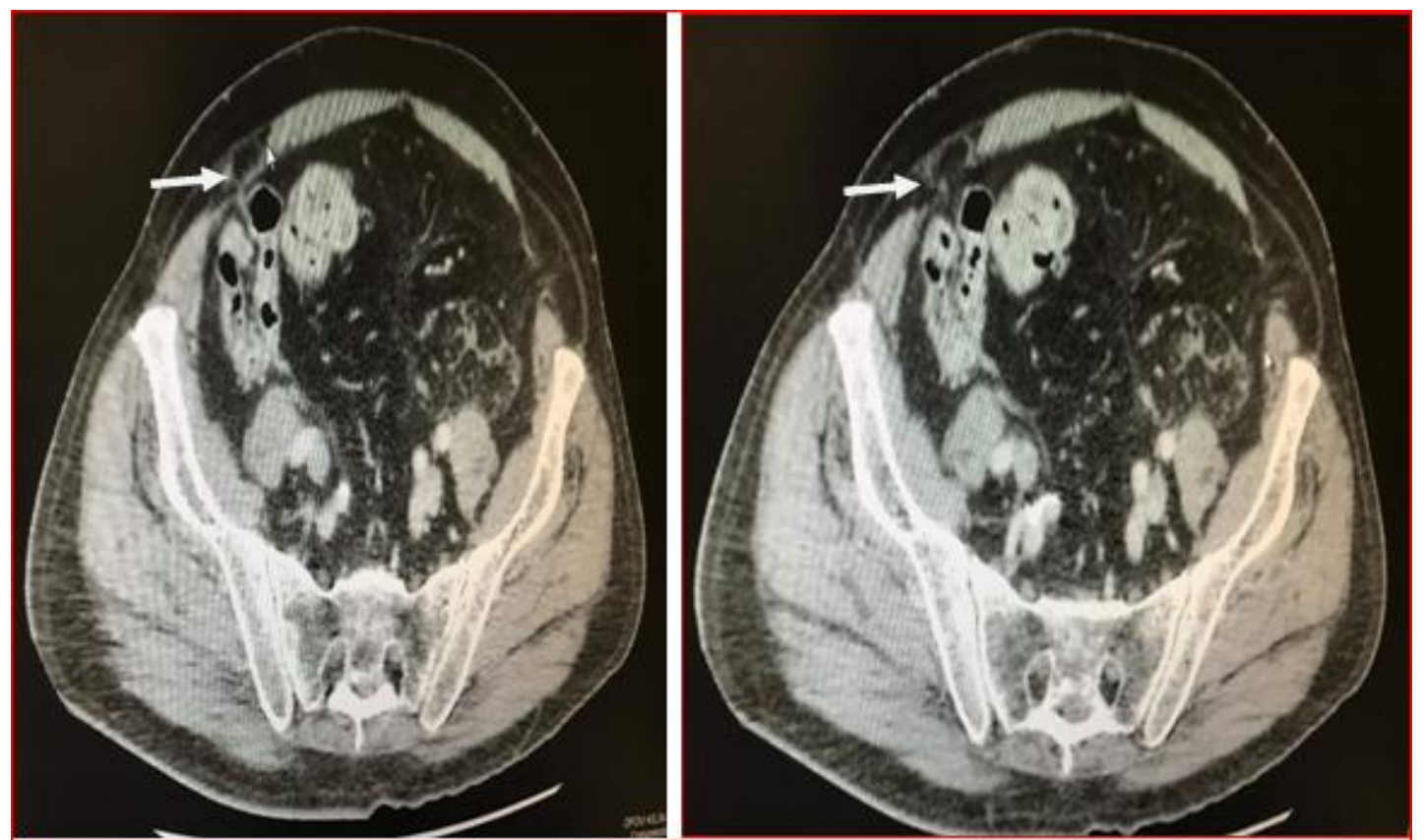

Fig-1: Abdominal CT scan revealing left Spigelian hernia (arrows)

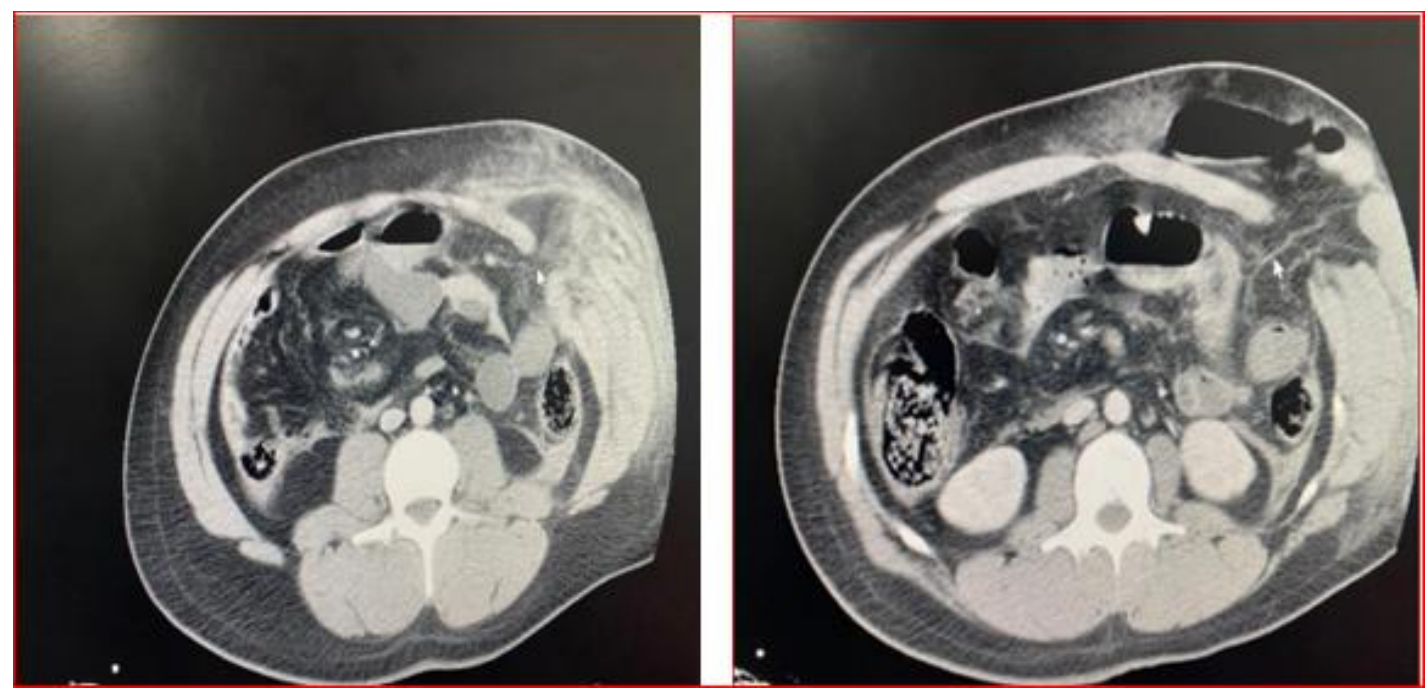

Fig-2: Abdominal CT scan revealing right sided post traumatic Spigelian hernia (arrows)

\section{DISCUSSION}

Named after Adrian van der Spiegel, an anatomist from Brussels, Spigelian hernias occur secondary to a defect in the transverses abdominis muscle and rectus sheath aponeurosis, which allows abdominal contents to protrude through the linea semilunaris (less commonly known as the Spigelian line or belt). Most occur justbelow the umbilicus where the aponeurosis is widest and weakest.Spigelian hernia contents most often includes small intestine, butcan also include cecum appendix, sigmoid colon or omentum [3, 4].
Spigelian hernia can be congenital as acquired (described in most publications). The musculoaponeurotic structure in the Spigelian fascia and ventral to it is generally considered to be the most important etiologic factor. Other factors are involved such as intra-abdomional pressure, obesity; some authors include the neurovascular openings in the Spigelian fascia may become enlarged, permitting herniation.

The clinical diagnosis of Spigelian hernia is challenging since the symptoms can be variable and non-specific.it is often made difficult by obesity, either when the hernia is small it is then barely palpable, or in the event of a large hernia protruding under the skin it 
can be mistaken for a lipoma as in the case of our observation or with an intra-abdominal tumor. It is estimated that around $50 \%$ of patients with spiegel hernia did not have a correct preoperative diagnosis [5]. Pain is the most common symptom and is usually more localized to that side of the abdomen.

Abdominal CT scan, with a sensitivity of $100 \%$ [6], remains the complementary examination of choice to dispel any diagnostic doubts, although the hernia might be apparent on ultrasound (US) or clinical exam in some patients [3], as it accurately demonstrates the parietal defect, the location and the contents of the sac [7] it allows also to indicate an urgent therapeutic management in case of presence of signs of strangulation.

Surgical treatment should be started as soon as possible because of the high risk of incarceration. The parietal repair by prosthetic mesh is the treatment of choice [8], the laparoscopic route in addition to its diagnostic interest had proven its effectiveness in the treatment of this type of hernia [9, 10], and the conventional route is still in order especially in large hernias

\section{CONCLUSION}

Spigelian hernia is a rare affection of young adult, the clinical diagnosis is sometimes difficult, but the abdominal CT scan remains very sensitive for confirmation of the diagnosis. The significant risk of strangulation requires surgical treatment once the diagnosis is confirmed.

\section{REFERENCES}

1. Spangen L. Spigelian hernia. World J Surg. 1989;13:573-80

2. Guivarch M, Boche O, Scherrer A, Roullet-Audy J.C. Hernies ventrales antérolatérales dites de Spigel. 51 cas. Bull Acad Natl Med. 2004;188:1041-54

3. Hiller N, Alberton Y, Shapira Y, Hadas-Halpern I. Richter's hernia strangulated in a Spigelian hernia: ultrasonic diagnosis. J Clin Ultrasound. 1994; 22: 503-5.

4. Miller R, Lifschitz O, Mavor E. Incarcerated Spigelian hernia mimicking obstructing colon carcinoma. Hernia. 2008;12:87-9.

5. Sabiston DC, Lyerly HK, Adams HG. Treatise on surgical Interamericana; 1999.

6. Light D, Chattopadhyay D, Bawa S. Radiological and clinical examination in the diagnosis of Spigelian hernias. The Annals of The Royal College of Surgeons of England. 2013 Mar;95(2):98-100.

7. Moles Morenilla L, Docobo Durántez F, Mena Robles J, Quinta Frutos RD. Hernia de Spiegel en España: Análisis de 162 casos. Revista Española de Enfermedades Digestivas. 2005 May;97(5):338-47.

8. Antonio Carbonell-Tatay, Francisco Javier Landete Molina. Hernia de Spiegel. ArchCir GenDig. 2004 Octubre

9. García-Molina F, Ortegón-Castellano B, FrancoOsorio J. D., Dominguez-Adame-Lama E, GilQuirós F

10. Kasirajan K, López J, López R. Laparoscopic technique in the management of spigelian hernia. J Laparoendosc Adv Surg Tech A. 1997 Dec; Dec;7(6):385. 